\title{
Four solutions of an inhomogeneous elliptic equation with critical exponent and singular term
}

\author{
Jianqing Chen ${ }^{a}$ Eugénio M. Rocha ${ }^{\mathrm{b}, *}$ \\ ${ }^{a}$ Department of Mathematics, Fujian Normal University, Fuzhou 35000\%, P.R. \\ China \\ ${ }^{\mathrm{b}}$ Department of Mathematics, University of Aveiro, 3810-193 Aveiro, Portugal
}

\section{Abstract}

In this paper, we prove the existence of four nontrivial solutions of

$$
-\Delta u-\frac{\lambda}{|x|^{2}} u=|u|^{2^{*}-2} u+\mu|x|^{\alpha-2} u+f(x), \quad x \in \Omega \backslash\{0\}
$$

and show that at least one of them is sign changing. Our results extend some previous works on the literature, as Tarantello(1993), Kang-Deng(2005) and HiranoShioji(2005).

Key words: Variational methods, inhomogeneous Laplacian equation, singular term, four solutions, sign changing solution.

2000 MSC: 35J20, 35J70.

\section{Introduction}

In Chen-Li-Li [4], it has been showed the effect of suitable singular potential $V(x)$ on the existence of multiple solutions of

$$
-\Delta u=\lambda V(x) u+|u|^{2^{*}-2} u, \quad u \in H_{0}^{1}(\Omega) .
$$

\footnotetext{
* Corresponding author.

Email addresses: jqchen@fjnu.edu.cn (Jianqing Chen), eugenio@ua.pt (Eugénio M. Rocha).
} 
Here, we will prove an additional inhomogeneous perturbation of (E) can produce more solutions. More precisely, we study the existence of four solutions of the following problem

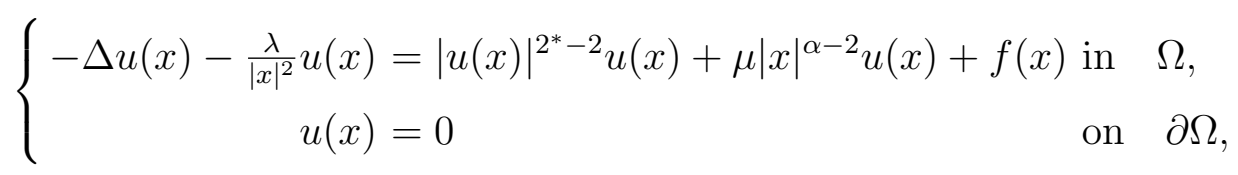

and we prove that at least one of them is sign changing. We assume that $0 \in$ $\Omega \subset \mathbb{R}^{N}(N \geq 3)$ is a bounded domain with smooth boundary, $2^{*} \doteq 2 N /(N-2)$ is the critical Sobolev exponent, $0 \leq \lambda<\Lambda \doteq((N-2) / 2)^{2}$ and $f \in L^{\infty}(\Omega)$.

Elliptic equations with a singular term have attracted great interests during the past several years, e.g. see Ferrero-Gazzola [8], Jannelli [10], Terracini [17], Smets [13]. Particularly, we point out that when $\alpha=2$ and $N \geq 7$, KangDeng [11] proved the existence of two nontrivial solutions of Eq.(1) provided $f$ satisfying some other conditions. The main result in this paper (Theorem 1.2) proves the existence of four solutions of Eq.(1), and also implies that suitable unbounded coefficients $|x|^{\alpha-2}$ can release the restriction of spatial dimension $N$.

In what follows, we state the main result (Theorem 1.2) but for the presentation coherence, we first prove an auxiliary result (Lemma 1.1). Assume that $\alpha>0$. From the work of Chaudhuri-Ramaswamy [2], we know that

$$
\mu_{1} \doteq \inf \left\{\int_{\Omega}\left(|\nabla u|^{2}-\frac{\lambda}{|x|^{2}}|u|^{2}\right) d x: \int_{\Omega}|x|^{\alpha-2}|u|^{2} d x=1\right\}>0 .
$$

Define $p \doteq 2^{*}, T(u) \doteq \int_{\Omega}\left(|\nabla u|^{2}-\frac{\lambda}{|x|^{2}}|u|^{2}-\mu|x|^{\alpha-2}|u|^{2}\right) d x$ and

$$
M \doteq \inf \left\{(T(u))^{\frac{1}{2}}: \int_{\Omega}|u|^{p} d x=1\right\} .
$$

Lemma 1.1 If $0 \leq \lambda<\Lambda, \alpha>0$ and $0<\mu<\mu_{1}$, then $M>0$.

Proof. For any $u \neq 0$, we have from the assumption $0<\mu<\mu_{1}$ and the Hardy inequality that

$$
T(u) \geq\left(1-\frac{\mu}{\mu_{1}}\right) \int_{\Omega}\left(|\nabla u|^{2}-\frac{\lambda}{|x|^{2}}|u|^{2}\right) d x \geq\left(1-\frac{\mu}{\mu_{1}}\right)\left(1-\frac{\lambda}{\Lambda}\right) \int_{\Omega}|\nabla u|^{2} d x .
$$

Thus

$$
\left(1-\frac{\mu}{\mu_{1}}\right)\left(1-\frac{\lambda}{\Lambda}\right) \int_{\Omega}|\nabla u|^{2} d x \leq T(u) \leq \int_{\Omega}|\nabla u|^{2} d x
$$

Note that the best Sobolev constant

$$
S_{0} \doteq \inf \left\{\int_{\Omega}|\nabla u|^{2} d x: \int_{\Omega}|u|^{p} d x=1\right\}>0 .
$$


We immediately have that $M>0$.

Throughout this paper, we always assume that $0 \leq \lambda<\Lambda$ and $0<\mu<\mu_{1}$. We say that $u \in H_{0}^{1}(\Omega)$ is a solution of Eq.(1) if and only if $u$ is a critical point of the Euler functional $I(u) \doteq \frac{1}{2} T(u)-\frac{1}{p} \int_{\Omega}|u|^{p} d x-\int_{\Omega} f u d x$, i.e. for any $v \in H_{0}^{1}(\Omega)$ there holds

$$
\int_{\Omega}\left(\nabla u \nabla v-\frac{\lambda}{|x|^{2}} u v-\mu|x|^{\alpha-2} u v-|u|^{p-2} u v-f v\right) d x=0 .
$$

We will prove the following result:

Theorem 1.2 Let $0 \leq \lambda<\Lambda, 0<\mu<\mu_{1}, f \in L^{\infty}(\Omega)$ and satisfies

$$
|f|_{\frac{p}{p-1}} \doteq|f|_{\sharp}<\frac{p-2}{2(p-1)} M\left(\frac{M^{p}}{p-1}\right)^{\frac{1}{p-2}} .
$$

If $0<\alpha<\sqrt{\Lambda-\lambda}$, then Eq.(1) has at least four nontrivial solutions in $H_{0}^{1}(\Omega)$ and at least one of them is sign changing.

The paper is organized as follows. In Section 2, we give some preliminaries. In Section 3, we obtain the necessary auxiliary results in order to prove the Theorem 1.2.

The proof of Theorem 1.2 is based on variational methods and it is inspired by Tarantello [15] and Hirano-Shioji [9]. The main strategy, based on four steps, is the following. In the first step, we define a Nehari type set $\mathscr{M}$ and use assumption (A) to divide $\mathscr{M}$ into three subsets $\mathscr{M}^{+}, \mathscr{M}^{0}$ and $\mathscr{M}^{-}$. In the second step, we solve two minimization problem $\inf _{\mathscr{M}} I$ and $\inf \mathscr{M}^{-} I$ and get two solutions $w_{0}, w_{1}$ of Eq.(1). In the third step, we construct two subsets $\mathscr{M}_{1}^{-}$ and $\mathscr{M}_{2}^{-}$of $\mathscr{M}^{-}$and prove that the minimizer of $\inf \left\{I(u): u \in \mathscr{M}_{1}^{-} \cap \mathscr{M}_{2}^{-}\right\}$ is a sign changing solution of Eq.(1). In the final step, we define a translated functional and get a fourth solution of Eq.(1).

Note that, although the proof is inspired by Tarantello [15] and Hirano-Shioji [9], the arguments used by them can not be directly applied here, since we are facing the singular term $\frac{\lambda}{|x|^{2}} u$ (see Remark 3.6, Remark 3.9 and Remark 3.12). In fact, we need to develop some techniques recently used in Chen $[5,6]$ and the exact local behavior for the solutions of Eq.(1), in order to overcome the difficulties created by the singular term $\frac{\lambda}{|x|^{2}} u$.

Notations. In what follows, we denote the norm in $H_{0}^{1}(\Omega)$ by $\|\cdot\|$, the integral $\int_{\Omega} \cdot d x$ by $\int \cdot$, and the ball in $\mathbb{R}^{N}$ with center at $x$ and radius $R$ by $B(x, R)$. We use $\doteq$ to emphasize a new definition. Different positive constants may be denoted by the same letter $K$ or $K_{j}$. Additionally, $O\left(\varepsilon^{\beta}\right)$ means 
that $\left|O\left(\varepsilon^{\beta}\right) \varepsilon^{-\beta}\right| \leq K, o\left(\varepsilon^{\beta}\right)$ is $\left|o\left(\varepsilon^{\beta}\right) \varepsilon^{-\beta}\right| \rightarrow 0$ as $\varepsilon \rightarrow 0$, and $o(1)$ is an infinitesimal value, and $\rightarrow$ (respectively, $\rightarrow$ ) will denote strongly (respectively, weakly) convergence.

\section{Preliminaries}

In this section, we give some preliminaries which play important roles in the variational methods used to solve Eq.(1). Namely, we briefly describe the solution of an auxiliary problem, the local behavior of the solutions of Eq.(1) and some integral estimates.

From Catrina-Wang [3], Terracini [17], and Chou-Chu [7], we have the following proposition:

Proposition 2.1 For $0<\lambda<\Lambda=\left(\frac{N-2}{2}\right)^{2}$, equation

$$
-\Delta u-\frac{\lambda}{|x|^{2}} u=|u|^{2^{*}-2} u \quad x \in \mathbb{R}^{N} \backslash\{0\}, \quad u(x) \rightarrow 0 \text { as }|x| \rightarrow+\infty,
$$

has a family of solutions

$$
U_{\varepsilon}(x)=\frac{[4 \varepsilon(\Lambda-\lambda) N /(N-2)]^{\frac{N-2}{4}}}{\left[\varepsilon|x|^{\gamma^{\prime} / \sqrt{\Lambda}}+|x|^{\gamma / \sqrt{\Lambda}}\right]^{\frac{N-2}{2}}}, \quad \varepsilon>0 .
$$

where $\Lambda=\left(\frac{N-2}{2}\right)^{2}, \gamma^{\prime}=\sqrt{\Lambda}-\sqrt{\Lambda-\lambda}, \gamma=\sqrt{\Lambda}+\sqrt{\Lambda-\lambda}$. Moreover $U_{\varepsilon}(x)$ is the unique positive radial symmetric solution of Eq.(2) up to a dilation. And $U_{\varepsilon}(x)$ is the extremal function of the minimization problem

$$
S_{\lambda}=\inf \left\{\int_{\mathbb{R}^{N}}\left(|\nabla u|^{2}-\frac{\lambda}{|x|^{2}} u^{2}\right) d x ; \quad u \in \mathcal{D}^{1,2}\left(\mathbb{R}^{N}\right), \int_{\mathbb{R}^{N}}|u|^{2^{*}} d x=1\right\} .
$$

Clearly,

$$
\int_{\mathbb{R}^{N}}\left|U_{\varepsilon}(x)\right|^{2^{*}} d x=\int_{\mathbb{R}^{N}}\left(\left|\nabla U_{\varepsilon}\right|^{2}-\frac{\lambda}{|x|^{2}} U_{\varepsilon}^{2}\right) d x=S_{\lambda}^{\frac{N}{2}}
$$

We now recall some exact local behavior of the solutions of Eq.(1) (see Chen $[5$, Th.1.1]).

Proposition 2.2 Let $0 \leq \lambda<\Lambda$. We have that

- if $u \in H_{0}^{1}(\Omega)$ is a solution of Eq.(1), then there holds

$$
|u(x)| \leq K_{1}|x|^{-(\sqrt{\Lambda}-\sqrt{\Lambda-\lambda})}, \quad x \in B(0, r) \backslash\{0\}
$$

for some positive constant $K_{1}$ and sufficiently small $r>0$; 
- if $u \in H_{0}^{1}(\Omega)$ is a positive solution of Eq.(1), then there holds

$$
K_{2}|x|^{-(\sqrt{\Lambda}-\sqrt{\Lambda-\lambda})} \leq|u(x)| \leq K_{1}|x|^{-(\sqrt{\Lambda}-\sqrt{\Lambda-\lambda})}, \quad x \in B(0, r) \backslash\{0\}
$$

for $r>0$ sufficiently small and some positive constants $K_{1}, K_{2}$.

Remark 2.3 Let u be a positive solution of Eq.(1).

(i) When $\lambda=0, u(0)$ is positive and we come back to the usual case.

(ii) When $0<\lambda<\Lambda$, the singular order at $x=0$ of $u$ stated in Proposition 2.2 coincide with the singularity of the explicit form $U_{\varepsilon}(x)$.

(iii) When $\lambda \rightarrow \Lambda$, the singularity of the positive solutions become more and more stronger.

The following integral estimates are also relevant. Define a cut-off function $\phi(x)=1$ if $|x| \leq \delta, \phi(x)=0$ if $|x| \geq 2 \delta, \phi(x) \in C_{0}^{1}(\Omega)$ and $|\phi(x)| \leq 1$, $|\nabla \phi(x)| \leq C$. Let $v_{\varepsilon}(x)=\phi(x) U_{\varepsilon}(x)$.

Proposition 2.4 Let $0 \leq \lambda<\Lambda$ and $w \in H_{0}^{1}(\Omega)$ be a solution of Eq.(1). Then for $\varepsilon>0$ small enough we have that

$$
\begin{aligned}
\int w^{2^{*}-1} v_{\varepsilon} & =O\left(\varepsilon^{\frac{N-2}{4}}\right) \quad \text { and } \int w v_{\varepsilon}^{2^{*}-1} d x=O\left(\varepsilon^{\frac{N-2}{4}}\right) ; \\
\int\left(\left|\nabla v_{\varepsilon}\right|^{2}-\frac{\lambda}{|x|^{2}} v_{\varepsilon}^{2}\right) & =S_{\lambda}^{\frac{N}{2}}+O\left(\varepsilon^{\frac{N}{2}}\right)+O\left(\varepsilon^{\frac{N-2}{2}}\right) ; \\
\int v_{\varepsilon}^{2^{*}} & =S_{\lambda}^{\frac{N}{2}}-O\left(\varepsilon^{\frac{N}{2}}\right) ; \\
\int|x|^{\alpha-2} v_{\varepsilon}^{2} & =O\left(\varepsilon^{\frac{\alpha \sqrt{\Lambda}}{2 \sqrt{\Lambda-\lambda}}}\right), \text { when } 0<\alpha<2 \sqrt{\Lambda-\lambda} ; \\
\int v_{\varepsilon} d x & =O\left(\varepsilon^{\frac{N-2}{4}}\right) ;
\end{aligned}
$$

Proof. For the proofs of (5), (6) and (7), see Chen [6]. Here we only prove (8) and (9). Recalling the definition of $v_{\varepsilon}$, we have that

$$
\begin{gathered}
\begin{aligned}
\int|x|^{\alpha-2} v_{\varepsilon}^{2} & =\int_{\Omega \backslash B(0, \delta)}|x|^{\alpha-2} v_{\varepsilon}^{2}+\int_{B(0, \delta)}|x|^{\alpha-2} v_{\varepsilon}^{2} \\
& =O\left(\varepsilon^{\frac{N-2}{2}}\right)+\int_{B(0, \delta)}|x|^{\alpha-2} v_{\varepsilon}^{2} \cdot
\end{aligned} \\
\int_{B(0, \delta)}|x|^{\alpha-2} v_{\varepsilon}^{2} d x=K \cdot \varepsilon^{\frac{(N-2)}{2}} \int_{0}^{\delta} \frac{\rho_{\alpha-2+N-1} d \rho}{\left[\varepsilon \rho^{\gamma^{\prime} / \sqrt{\Lambda}}+\rho^{\gamma / \sqrt{\Lambda}}\right]^{N-2}} \\
=K \cdot \varepsilon^{\frac{(N-2)}{2}} \int_{0}^{\delta} \frac{\rho_{\alpha-2+N-1} d \rho}{\varepsilon^{N-2} \rho^{2 \gamma^{\prime}}\left[1+\varepsilon^{-1} \rho^{2 \sqrt{\Lambda-\lambda} / \sqrt{\Lambda}}\right]^{N-2}} \\
=K \cdot \varepsilon^{\frac{(N-2)}{2}} \int_{0}^{\delta \varepsilon^{-\frac{\sqrt{\Lambda}}{2 \sqrt{\Lambda-\lambda}}}} \frac{\varepsilon^{\frac{(\alpha-2+N) \sqrt{\Lambda}}{2 \sqrt{\Lambda-\lambda}}} \rho_{\alpha-2+N-1} d \rho}{\varepsilon^{N-2} \varepsilon^{\frac{\gamma^{\prime}}{2 \sqrt{\Lambda-\lambda}}} \rho^{2 \gamma^{\prime}}\left[1+\rho^{2 \sqrt{\Lambda-\lambda} / \sqrt{\Lambda}}\right]^{N-2}}
\end{gathered}
$$


Since

$$
\begin{gathered}
-1+\alpha-2+N-2(\sqrt{\Lambda}-\sqrt{\Lambda-\lambda})-4 \sqrt{\Lambda-\lambda}=-1+\alpha-2 \sqrt{\Lambda-\lambda}<-1, \\
\frac{(\alpha-2+N) \sqrt{\Lambda}}{2 \sqrt{\Lambda-\lambda}}-(N-2)-\frac{\gamma^{\prime} \sqrt{\Lambda}}{\sqrt{\Lambda-\lambda}}=\frac{\alpha \sqrt{\Lambda}}{2 \sqrt{\Lambda-\lambda}}-\sqrt{\Lambda}<0,
\end{gathered}
$$

we get that

$$
\int_{B(0, \delta)}|x|^{\alpha-2} v_{\varepsilon}^{2} d x=K \cdot \varepsilon^{\frac{\alpha \sqrt{\Lambda}}{2 \sqrt{\Lambda-\lambda}}} .
$$

It follows from $\int_{\Omega \backslash B(0, \delta)}|x|^{\alpha-2} v_{\varepsilon}^{2}=O\left(\varepsilon^{\frac{N-2}{2}}\right)$ and $0<\alpha<2 \sqrt{\Lambda-\lambda}$ that

$$
\int|x|^{\alpha-2} v_{\varepsilon}^{2}=O\left(\varepsilon^{\frac{\alpha \sqrt{\Lambda}}{2 \sqrt{\Lambda-\lambda}}}\right), \text { for } \varepsilon>0 \text { small enough. }
$$

This proves (8). The proof of (9) is similar but simpler than the proof of (8), so we omit the details.

\section{Proof of Theorem 1.2}

In this section, we will prove Theorem 1.2. As described in the Introduction, the proof is divided into four steps. We start defining some Nehari type sets; we prove the existence of two solutions of Eq.(1); we prove the existence of a third solution which is a sign changing solution of Eq.(1); and we prove the existence of a fourth solution by a translated argument.

Firstly for $u \in H_{0}^{1}(\Omega)$, recall the definition of

$$
T(u) \doteq \int\left(|\nabla u|^{2}-\frac{\lambda}{|x|^{2}}|u|^{2}-\mu|x|^{\alpha-2}|u|^{2}\right) .
$$

So, the Euler functional can be rewritten as $I(u)=\frac{1}{2} T(u)-\frac{1}{p} \int|u|^{p}-\int f u$. We define

$$
Q(u) \doteq T(u)-\int|u|^{p}-\int f u \quad \text { and } \quad J(u) \doteq 2 T(u)-p \int|u|^{p}-\int f u,
$$

then $Q$ and $J$ are well defined $C^{1}$ functionals on $H_{0}^{1}(\Omega)$. Next, set

$$
\mathscr{M} \doteq\left\{u \in H_{0}^{1}(\Omega): Q(u)=0\right\} .
$$

Then for any $u \in \mathscr{M}, J(u)=T(u)-(p-1) \int|u|^{p}$. We also define several subsets of $\mathscr{M}$,

$$
\mathscr{M}^{+} \doteq\{u \in \mathscr{M}: J(u)>0\}, \quad \mathscr{M}^{0} \doteq\{u \in \mathscr{M}: J(u)=0\}
$$


and $\mathscr{M}^{-} \doteq\{u \in \mathscr{M}: J(u)<0\}$.

Lemma 3.1 Let $0 \leq \lambda<\Lambda$ and $0<\mu<\mu_{1}$. Then the following hold:

(i) $(p-2) T(u)^{\frac{1}{2}}<(p-1) M^{-1}|f|_{\sharp}$ for all $u \in \mathscr{M}^{+}$;

(ii) $T(u)>\left(\frac{M^{p}}{p-1}\right)^{\frac{2}{p-2}}$ for all $u \in \mathscr{M}^{-}$;

(iii) if (A) holds, then $I(u) \geq 0$ for all $u \in \mathscr{M}^{-}$.

Proof. (i) For any $u \in \mathscr{M}^{+}$, using $Q(u)=0$ we get that

$$
\begin{aligned}
0<J(u) & =T(u)-(p-1) \int|u|^{p}=T(u)-(p-1)\left(T(u)-\int f u\right) \\
& =(2-p) T(u)+(p-1) \int f u .
\end{aligned}
$$

Since $M|u|_{p} \leq T(u)^{\frac{1}{2}}$, we get that

$$
(p-2) T(u)<(p-1)|f|_{\sharp}|u|_{p} \leq(p-1)|f|_{\sharp} M^{-1} T(u)^{\frac{1}{2}} .
$$

Therefore

$$
(p-2) T(u)^{\frac{1}{2}}<(p-1) M^{-1}|f|_{\sharp} .
$$

(ii) For any $u \in \mathscr{M}^{-}$, we have that $J(u)<0$. From the definition of $M$, we have that $\int|u|^{p} \leq M^{-p} T(u)^{\frac{p}{2}}$. Therefore

$$
T(u)<(p-1) \int|u|^{p} \leq(p-1) M^{-p} T(u)^{\frac{p}{2}}
$$

Thus

$$
T(u)>\left(\frac{M^{p}}{p-1}\right)^{\frac{2}{p-2}}
$$

(iii) For any $u \in \mathscr{M}^{-} \subset \mathscr{M}$, we obtain from $\int|u|^{p}=T(u)-\int f u$ that

$$
\begin{aligned}
I(u) & =\frac{1}{2} T(u)-\frac{1}{p}\left(T(u)-\int f u\right)-\int f u \\
& =\frac{p-2}{2 p} T(u)-\frac{p-1}{p} \int f u \\
& \geq \frac{p-2}{2 p} T(u)-\frac{p-1}{p}|f|_{\sharp}|u|_{p} \\
& \geq \frac{p-2}{2 p} T(u)-\frac{p-1}{p}|f|_{\sharp} M^{-1} T(u)^{\frac{1}{2}} \\
& =\frac{1}{p} T(u)^{\frac{1}{2}}\left(\frac{p-2}{2} T(u)^{\frac{1}{2}}-(p-1)|f|_{\sharp} M^{-1}\right) .
\end{aligned}
$$


On the other hand we get from (ii) that

$$
T(u)>\left(\frac{M^{p}}{p-1}\right)^{\frac{2}{p-2}} .
$$

It is deduced from (10) and (11) that

$$
I(u) \geq \frac{1}{p} T(u)^{\frac{1}{2}}\left(\frac{p-2}{2}\left(\frac{M^{p}}{p-1}\right)^{\frac{1}{p-2}}-(p-1)|f|_{\sharp} M^{-1}\right) .
$$

Therefore if $(\mathrm{A})$ holds, then $I(u) \geq 0$. The proof is complete.

Lemma 3.2 Let $0 \leq \lambda<\Lambda, 0<\mu<\mu_{1}$ and $f \not \equiv 0$ satisfy (A). Then for any $u \in H_{0}^{1}(\Omega)$ and $u \neq 0$, there exists a unique $t^{+}=t^{+}(u)>0$ such that $t^{+}(u) u \in \mathscr{M}^{-}$and

$$
t^{+}>\left(\frac{T(u)}{(p-1) \int|u|^{p}}\right)^{\frac{1}{p-2}} \doteq t_{\max }
$$

and

$$
I\left(t^{+} u\right)=\max _{t \geq t_{\max }} I(t u) .
$$

Moreover if $\int f u>0$, then there exists an unique $t^{-}=t^{-}(u)>0$ such that $t^{-}(u) u \in \mathscr{M}^{+}, t^{-}<t_{\max }$ and

$$
I\left(t^{-} u\right)=\inf _{0 \leq t \leq t_{\max }} I(t u) .
$$

Proof. The proof follows exactly the scheme in the proof of Lemma 2.1 in Tarantello [15].

Proposition 3.3 Assume $0 \leq \lambda<\Lambda, 0<\mu<\mu_{1}$ and (A) hold. Let $\left\{u_{n}\right\} \subset$ $\mathscr{M}^{-}$be such that $u_{n} \rightarrow u$ weakly in $H_{0}^{1}(\Omega)$ and $I\left(u_{n}\right) \rightarrow c$ but $u_{n}$ does not converge strongly to $u$ in $H_{0}^{1}(\Omega)$. Then the following holds:

(1) $c>I\left(t^{+}(u) u\right)$ in the case $u \neq 0$ and $t^{+}(u) \leq 1$;

(2) $c \geq I\left(t^{-}(u) u\right)+\frac{1}{N} S_{\lambda}^{\frac{N}{2}}$ in the case $u \neq 0$ and $t^{+}(u)>1$;

(3) $c \geq \frac{1}{N} S_{\lambda}^{\frac{N}{2}}$ in the case $u=0$.

Proof. We use the methods employed in Hirano-Shioji [9]. Note that $u_{n} \rightarrow u$, $\int|x|^{\alpha-2}\left|u_{n}-u\right|^{2} \rightarrow 0$ as $n \rightarrow \infty$. We may assume that

$$
T\left(u_{n}-u\right)=\int\left(\left|\nabla u_{n}-\nabla u\right|^{2}-\frac{\lambda}{|x|^{2}}\left|u_{n}-u\right|^{2}\right)+0(1) \rightarrow a^{2}
$$




$$
\text { and } \quad \int\left|u_{n}-u\right|^{p} \rightarrow b^{p}
$$

Since $u_{n}$ does not converge strongly to $u$, we have $a \neq 0$. We set

$$
r(t)=I(t u), \quad \beta(t)=\frac{a^{2}}{2} t^{2}-\frac{b^{p}}{p} t^{p}
$$

and $\theta(t)=r(t)+\beta(t)$, then $I\left(t u_{n}\right) \rightarrow \theta(t)$ as $n \rightarrow+\infty$. We consider three situations:

(1) When $u \neq 0$ and $t^{+}(u) \leq 1$. Recall, that $t^{+}(u)$ is defined according to Lemma 3.2. We have that $r^{\prime}(1) \leq 0$. Since $u_{n} \in \mathscr{M}^{-}$, we have that $\theta^{\prime}(1)=0$. Thus $\beta^{\prime}(1) \geq 0$ and hence $a^{2}-b^{p} \geq 0$. So we have that $\beta\left(t^{+}(u)\right)>0$ and hence

$$
c \geq \theta(1) \geq \theta\left(t^{+}(u)\right)=I\left(t^{+}(u) u\right)+\beta\left(t^{+}(u)\right)>I\left(t^{+}(u) u\right) .
$$

(2) When $u \neq 0$ and $t^{+}(u)>1$. We have first from $t^{+}(u)>1$ that $b \neq 0$. Indeed if $b=0$, then from $\theta^{\prime}(1)=0$ and $\theta^{\prime \prime}(1) \leq 0$, we have that $r^{\prime}(1)=-a^{2}<0$ and $r^{\prime \prime}(1) \leq-a^{2}<0$, which contradicts to $t^{+}(u)>1$. So we have $b \neq 0$. We set $t_{*}=\left(a^{2} / b^{p}\right)^{\frac{1}{p-2}}$. We know that $\beta$ attains its maximum at $t_{*}$ and $\beta^{\prime}(t)>0$ for $0<t<t_{*}$ and $\beta^{\prime}(t)<0$ for $t>t_{*}$. Therefore we have that $\beta\left(t_{*}\right)=\left(a^{2} / b^{2}\right)^{\frac{N}{2}} / N \geq \frac{1}{N} S_{\lambda}^{\frac{N}{2}}$. Next, we will show that $t_{*} \leq t^{+}(u)$. Suppose this is not the case, i.e., $1<t^{+}(u)<t_{*}$. As $0>\theta^{\prime}(t)=r^{\prime}(t)+\beta^{\prime}(t)$ for all $t>1$, we have $r^{\prime}(t) \leq-\beta^{\prime}(t)<0$ for $t \in\left(1, t_{*}\right)$, which contradicts to $1<t^{+}(u)<t_{*}$ and $r^{\prime}\left(t^{+}(u)\right)=0$. So we have shown that $t_{*} \leq t^{+}(u)$. Hence we obtain

$$
c=\theta(1) \geq \theta\left(t_{*}\right)=I\left(t_{*} u\right)+\beta\left(t_{*}\right) \geq I\left(t^{-}(u) u\right)+\frac{1}{N} S_{\lambda}^{\frac{N}{2}} .
$$

This implies that (ii) holds.

(3) When $u=0$. Since $u_{n} \in \mathscr{M}^{-} \subset \mathscr{M}$, we have that

$$
\int\left(\left|\nabla u_{n}\right|^{2}-\frac{\lambda}{|x|^{2}}\left|u_{n}\right|^{2}\right)=\int\left|u_{n}\right|^{p}+0(1)
$$

Using the fact that $S_{\lambda}|v|_{p}^{2} \leq \int\left(|\nabla v|^{2}-\frac{\lambda}{|x|^{2}}|v|^{2}\right)$ for all $v \in H_{0}^{1}(\Omega)$ and $v \neq 0$, we obtain that

$$
\begin{aligned}
c & \geq \frac{1}{2} \int\left(\left|\nabla u_{n}\right|^{2}-\frac{\lambda}{|x|^{2}}\left|u_{n}\right|^{2}\right)-\frac{1}{p} \int\left|u_{n}\right|^{p}+0(1) \\
& \geq\left(\frac{1}{2}-\frac{1}{p}\right) \int\left(\left|\nabla u_{n}\right|^{2}-\frac{\lambda}{|x|^{2}}\left|u_{n}\right|^{2}\right)+0(1) \geq \frac{1}{N} S_{\lambda}^{\frac{N}{2}} .
\end{aligned}
$$

The proof is complete. 


\subsection{Existence of two solutions}

In this subsection, we prove the existence of two solutions of Eq.(1). Set

$$
c_{0} \doteq \inf _{u \in \mathscr{M}^{\prime}} I(u) \quad \text { and } \quad c_{1} \doteq \inf _{u \in \mathscr{M}^{-}} I(u)
$$

Proposition 3.4 If (A) holds, then $c_{0}<0$ and there is a critical point $w_{0} \in$ $\mathscr{M}^{+}$of $I$ such that $I\left(w_{0}\right)=c_{0}$ and $w_{0}$ is a local minimizer for $I$.

Proof. The proof is exactly the same as Tarantello [15]. We omit the details here.

Lemma 3.5 Let $0 \leq \lambda<\Lambda$ and $0<\alpha<\sqrt{\Lambda-\lambda}$. Then $c_{1}<c_{0}+\frac{1}{N} S_{\lambda}^{\frac{N}{2}}$.

Proof. First using the same argument as Tarantello [15, Proposition 2.2], we know that there is $s_{0}>0$ and $\varepsilon>0$ sufficiently small such that $w_{0}+$ $s_{0} v_{\varepsilon} \in \mathscr{M}^{-}$. Next to prove $c_{1}<c_{0}+\frac{1}{N} S_{\lambda}^{\frac{N}{2}}$, we only need to prove that $\sup _{s>0} I\left(w_{0}+s v_{\varepsilon}\right)<c_{0}+\frac{1}{N} S_{\lambda}^{\frac{N}{2}}$. Note that $I\left(w_{0}+s v_{\varepsilon}\right) \rightarrow-\infty$ as $s \rightarrow+\infty$, we only estimate $I\left(w_{0}+s v_{\varepsilon}\right)$ for bounded $s$. Since $w_{0}$ is a solution of Eq.(1), we have that

$$
\int\left(\nabla w_{0} \nabla v_{\varepsilon}-\frac{\lambda}{|x|^{2}} w_{0} v_{\varepsilon}-\mu|x|^{\alpha-2} w_{0} v_{\varepsilon}\right)=\int\left(w_{0}^{p-1} v_{\varepsilon}+f v_{\varepsilon}\right)
$$

Hence

$$
\begin{aligned}
I\left(w_{0}+s v_{\varepsilon}\right)= & \frac{1}{2} T\left(w_{0}+s v_{\varepsilon}\right)-\frac{1}{p} \int\left|w_{0}+s v_{\varepsilon}\right|^{p}-\int f\left(w_{0}+s v_{\varepsilon}\right) \\
= & I\left(w_{0}\right)+I\left(s v_{\varepsilon}\right)+2 s \int\left(w_{0}^{p-1} v_{\varepsilon}+f v_{\varepsilon}\right) \\
& \quad+\frac{1}{p} \int\left(w_{0}^{p}+\left|s v_{\varepsilon}\right|^{p}-\left|w_{0}+s v_{\varepsilon}\right|^{p}\right) \\
\leq & I\left(w_{0}\right)+I\left(s v_{\varepsilon}\right)+C \int\left(v_{\varepsilon}+w_{0}^{p-1} v_{\varepsilon}+w_{0} v_{\varepsilon}^{p-1}\right) .
\end{aligned}
$$

Note that

$$
\begin{aligned}
\sup _{s>0} I\left(s v_{\varepsilon}\right) & =\sup _{s>0}\left(\frac{s^{2}}{2} T\left(v_{\varepsilon}\right)-\frac{s^{p}}{p} \int v_{\varepsilon}^{p}-s \int f v_{\varepsilon}\right) \\
& \leq \sup _{s>0}\left(\frac{s^{2}}{2} T\left(v_{\varepsilon}\right)-\frac{s^{p}}{p} \int v_{\varepsilon}^{p}\right)+C \int v_{\varepsilon} \\
& \leq \frac{1}{N} T\left(v_{\varepsilon}\right)^{\frac{N}{2}}\left(\int v_{\varepsilon}^{p}\right)^{1-\frac{N}{2}}+C \int v_{\varepsilon} .
\end{aligned}
$$


We obtain from Proposition 2.4 that

$$
\begin{aligned}
\sup _{s>0} I\left(w_{0}+s v_{\varepsilon}\right)< & \frac{1}{N}\left(S_{\lambda}^{\frac{N}{2}}+O\left(\varepsilon^{\frac{N-2}{2}}\right)-O\left(\varepsilon^{\frac{\alpha \sqrt{\Lambda}}{2 \sqrt{\Lambda-\lambda}}}\right)\right)^{\frac{N}{2}}\left(S_{\lambda}^{\frac{N}{2}}-O\left(\varepsilon^{\frac{N}{2}}\right)\right)^{1-\frac{N}{2}} \\
& +c_{0}+O\left(\varepsilon^{\frac{N-2}{4}}\right) \\
= & \frac{1}{N} S_{\lambda}^{\frac{N}{2}}\left(1-O\left(\varepsilon^{\frac{\alpha \sqrt{\Lambda}}{2 \sqrt{\Lambda-\lambda}}}\right)\right)^{\frac{N}{2}}\left(1-O\left(\varepsilon^{\frac{N}{2}}\right)\right)^{1-\frac{N}{2}}+c_{0}+O\left(\varepsilon^{\frac{N-2}{4}}\right) \\
= & c_{0}+O\left(\varepsilon^{\frac{N-2}{4}}\right)+\frac{1}{N} S_{\lambda}^{\frac{N}{2}}-O\left(\varepsilon^{\frac{\alpha \sqrt{\Lambda}}{2 \sqrt{\Lambda-\lambda}}}\right) \\
< & c_{0}+\frac{1}{N} S_{\lambda}^{\frac{N}{2}} \text { since } \quad \alpha<\sqrt{\Lambda-\lambda} .
\end{aligned}
$$

The proof is complete.

Remark 3.6 We emphasize that in the estimate $\int w_{0}^{p-1} v_{\varepsilon}$ and $\int w_{0} v_{\varepsilon}^{p-1}$, the exact local behavior of the solution of Eq.(1) (see Propositions 2.2 and 2.4) played an essential role. Indeed, without Propositions 2.2, estimates $\int w_{0}^{p-1} v_{\varepsilon}$ and $\int w_{0} v_{\varepsilon}^{p-1}$ seem to be impossible.

Proposition 3.7 Let $0 \leq \lambda<\Lambda, 0<\mu<\mu_{1}$ and $0<\alpha<\sqrt{\Lambda-\lambda}$. If $f \in L^{\infty}(\Omega)$ and satisfies $(A)$, then there is a critical point $w_{1} \in \mathscr{M}^{-}$of I such that $I\left(w_{1}\right)=c_{1}$.

Proof. First we will prove that there is $w_{1} \in \mathscr{M}^{-}$of $I$ such that $I\left(w_{1}\right)=c_{1}$. Let $\left\{u_{n}\right\} \subset \mathscr{M}^{-}$and $I\left(u_{n}\right) \rightarrow c_{1}$. Then by direct calculations we know that

$$
0<\inf T\left(u_{n}\right) \leq \sup T\left(u_{n}\right)<\infty
$$

The definition of $\mu_{1}$ and $0<\mu<\mu_{1}$ implies that $\left\{u_{n}\right\}$ is bounded in $H_{0}^{1}(\Omega)$. We may assume that $\left\{u_{n}\right\}$ converges weakly to some $w_{1}$. By Proposition 3.3 we have that $w_{1} \neq 0$. Now suppose that $\left\{u_{n}\right\}$ does not converge to $w_{1}$. Then by (i) and (ii) of Proposition 3.3, we get that $c_{1}>I\left(t^{+}\left(w_{1}\right) w_{1}\right)$ or $c_{1} \geq$ $I\left(t^{-}\left(w_{1}\right) w_{1}\right)+\frac{1}{N} S_{\lambda}^{\frac{N}{2}} \geq c_{0}+\frac{1}{N} S_{\lambda}^{\frac{N}{2}}$. In any case we get a contradiction since $c_{1}<c_{0}+\frac{1}{N} S_{\lambda}^{\frac{N}{2}}$. Therefore $\left\{u_{n}\right\}$ converges strongly to $w_{1}$. This means $w_{1} \in \mathscr{M}^{-}$ and $I\left(w_{1}\right)=c_{1}$.

Next we will show that such $w_{1}$ is a weak solution of Eq.(1). Choose any $v \in H_{0}^{1}(\Omega)$. For any $\rho \in(0,1)$ we set $t_{\rho}=t^{+}\left(w_{1}+\rho v\right)$ (where $t^{+}\left(w_{1}+\rho v\right)$ is defined according to Lemma 3.2). Since $w_{1}, t_{\rho}\left(w_{1}+\rho v\right) \in \mathscr{M}^{-}$and $I\left(w_{1}\right)=$ $\inf _{u \in \mathscr{M}^{-}} I(u)$, we have that

$$
I\left(t_{\rho}\left(w_{1}+\rho v\right)\right) \geq I\left(w_{1}\right) .
$$


On the other hand from $w_{1} \in \mathscr{M}^{-}$, we have that for any $t>0, I\left(w_{1}\right) \geq I\left(t w_{1}\right)$. In particular, $I\left(w_{1}\right) \geq I\left(t_{\rho} w_{1}\right)$. Thus we have for any $\rho \in(0,1)$,

$$
I\left(t_{\rho}\left(w_{1}+\rho v\right)\right) \geq I\left(t_{\rho} w_{1}\right) .
$$

Hence we get that

$$
\begin{aligned}
0 \leq & \frac{1}{\rho}\left(I\left(t_{\rho}\left(w_{1}+\rho v\right)\right)-I\left(t_{\rho} w_{1}\right)\right) \\
= & \frac{1}{\rho} \frac{t_{\rho}^{2}}{2}\left(T\left(w_{1}+\rho v\right)-T\left(w_{1}\right)\right)-\frac{1}{\rho} \frac{t_{\rho}^{p}}{p} \int\left(\left|w_{1}+\rho v\right|^{p}-\left|w_{1}\right|^{p}\right) \\
& \quad \frac{1}{\rho} t_{\rho} \int\left(f\left(w_{1}+\rho v\right)-f w_{1}\right) .
\end{aligned}
$$

Since $t_{\rho} \rightarrow 1$ as $\rho \rightarrow 0+$, letting $\rho \rightarrow 0+$, we obtain

$$
\int\left(\nabla w_{1} \nabla v-\frac{\lambda}{|x|^{2}} w_{1} v-\mu|x|^{\alpha-2} w_{1} v-\left|w_{1}\right|^{p-2} w_{1} v-f v\right) \geq 0 .
$$

As $v$ is arbitrarily, we get that

$$
\int\left(\nabla w_{1} \nabla v-\frac{\lambda}{|x|^{2}} w_{1} v-\mu|x|^{\alpha-2} w_{1} v-\left|w_{1}\right|^{p-2} w_{1} v-f v\right)=0 .
$$

Which means that $w_{1}$ is a solution of Eq.(1).

\subsection{Existence of sign changing solution}

This subsection is devoted to proving the existence of sign changing solution of Eq.(1). For $u \in H_{0}^{1}(\Omega)$, denote $u^{+}=\max \{0, u\}$ and $u^{-}=\max \{0,-u\}$, then $u^{+}, u^{-} \in H_{0}^{1}(\Omega)$ and $u=u^{+}-u^{-}$. Following Tarantello [15], we define

$$
\mathscr{M}_{1}^{-} \doteq\left\{u \in \mathscr{M} ; \quad u^{+} \in \mathscr{M}^{-}\right\} \quad \text { and } \quad \mathscr{M}_{2}^{-} \doteq\left\{u \in \mathscr{M} ; \quad-u^{-} \in \mathscr{M}^{-}\right\} .
$$

Set

$$
\mathscr{M}_{*}^{-} \doteq \mathscr{M}_{1}^{-} \cap \mathscr{M}_{2}^{-}
$$

Lemma 3.8 Let $0 \leq \lambda<\Lambda$ and $0<\alpha<\sqrt{\Lambda-\lambda}$ and (A) holds. Then $\mathscr{M}_{*}^{-} \neq \emptyset$.

Proof. From the definition of $\mathscr{M}_{*}^{-}$, we only need to prove that there exist $s>0$ and $t \in \mathbb{R}$ such that

$$
s\left(w_{1}-t U_{\varepsilon}\right)^{+} \in \mathscr{M}^{-} \text {and } \quad-s\left(w_{1}-t U_{\varepsilon}\right)^{-} \in \mathscr{M}^{-} .
$$


To this purpose, let

$$
t_{2} \doteq \max _{\bar{\Omega} \backslash\{0\}} \frac{w_{1}}{U_{\varepsilon}} \quad \text { and } \quad t_{1} \doteq \min _{\bar{\Omega} \backslash\{0\}} \frac{w_{1}}{U_{\varepsilon}}
$$

For $t \in\left(t_{1}, t_{2}\right),\left(w_{1}-t U_{\varepsilon}\right)^{+}$and $-\left(w_{1}-t U_{\varepsilon}\right)^{-}$, denoted by $s^{+}(t)$ and $s^{-}(t)$ the positive values given by Lemma 3.2 , according to which we have

$$
s^{+}(t)\left(w_{1}-t U_{\varepsilon}\right)^{+} \in \mathscr{M}^{-} \quad \text { and } \quad-s^{-}(t)\left(w_{1}-t U_{\varepsilon}\right)^{-} \in \mathscr{M}^{-} .
$$

Note that $s^{+}(t)$ is continuous with respect to $t$ satisfying

$$
\lim _{t \rightarrow t_{1}+0} s^{+}(t)=t^{+}\left(w_{1}-t_{1} U_{\varepsilon}\right)<+\infty \quad \text { and } \quad \lim _{t \rightarrow t_{2}-0} s^{+}(t)=+\infty .
$$

Similarly, $s^{-}(t)$ is continuous with respect to $t$ and

$$
\lim _{t \rightarrow t_{1}+0} s^{-}(t)=+\infty \text { and } \quad \lim _{t \rightarrow t_{2}-0} s^{-}(t)=t^{+}\left(t_{2} U_{\varepsilon}-w_{1}\right)<+\infty .
$$

The continuity of $s^{+}(t)$ and $s^{-}(t)$ implies that there is $t_{0} \in\left(t_{1}, t_{2}\right)$ such that

$$
s^{+}\left(t_{0}\right)=s^{-}\left(t_{0}\right)=s_{0}>0 \text {. }
$$

This proves the Lemma.

Remark 3.9 The exact local behavior for the solution $w_{1}$ of Eq.(1) stated in Proposition 2.2 is essential in the definition of $t_{2}$ and $t_{1}$. Indeed because of Proposition 2.2 , both $t_{1}$ and $t_{2}$ are finite. It seems to be very difficult to prove this Lemma without Proposition 2.2.

Lemma 3.10 If (A) holds, then $\mathscr{M}_{1}^{-}, \mathscr{M}_{2}^{-} \subset \mathscr{M}^{-}$.

Proof. Let $u \in \mathscr{M}_{1}^{-}$, i.e. $u \in \mathscr{M}$ and $u^{+} \in \mathscr{M}^{-}$. Then we obtain from $T(u)-\int|u|^{p}=\int f u$ that

$$
\begin{aligned}
J(u) & =T(u)-(p-1) \int|u|^{p}=T(u)-(p-1)\left(T(u)-\int f u\right) \\
& =-(p-2) T(u)+(p-1) \int f u \\
& \leq-(p-2) T(u)+(p-1)|f|_{\sharp}|u|_{p} .
\end{aligned}
$$

By the definition of $M$, we get that $|u|_{p} \leq M^{-1} T(u)^{\frac{1}{2}}$. Therefore

$$
J(u) \leq-T(u)^{\frac{1}{2}}\left((p-2) T\left(u^{+}\right)^{\frac{1}{2}}-(p-1)|f|_{\sharp} M^{-1}\right)<0 .
$$


Now according to $u^{+} \in \mathscr{M}^{-}$and (2) of Lemma 3.1, $T\left(u^{+}\right)>\left(\frac{M^{p}}{p-1}\right)^{\frac{2}{p-2}}$. Combining this with assumption $(\mathrm{A}),(p-2) T\left(u^{+}\right)^{\frac{1}{2}}-(p-1)|f|_{\sharp} M^{-1}>0$. Thus $J(u)<0$ and hence $u \in \mathscr{M}^{-}$. This proves that $\mathscr{M}_{1}^{-} \subset \mathscr{M}^{-}$. By a similar argument we can prove that $\mathscr{M}_{2}^{-} \subset \mathscr{M}^{-}$.

Define

$$
c_{2} \doteq \inf _{u \in \mathscr{M}_{*}^{-}} I(u)
$$

Lemma 3.11 Let $0 \leq \lambda<\Lambda$ and $0<\alpha<\sqrt{\Lambda-\lambda}$ and (A) holds. Then $c_{2}<c_{1}+\frac{1}{N} S_{\lambda}^{\frac{N}{2}}$.

Proof. It suffices to estimate $I\left(s w_{1}-t U_{\varepsilon}\right)$ for $s \geq 0$ and $t \in \mathbb{R}$. Since at this time, $\varepsilon$ can be sufficiently small, we replace $U_{\varepsilon}$ by $v_{\varepsilon}=\phi(x) U_{\varepsilon}$ defined in Section 2. From the structure of $I$, we find there is $R>0$ possibly large such that $I\left(s w_{1}-t v_{\varepsilon}\right) \leq c_{1}$ for all $s^{2}+t^{2} \geq R^{2}$. Thus it suffices to estimate $I\left(s w_{1}-t v_{\varepsilon}\right)$ for all $s^{2}+t^{2} \leq R^{2}$. From an elementary inequality

$$
|c+d|^{q} \geq|c|^{q}+|d|^{q}-K\left(|c|^{q-1}|d|+|c||d|^{q-1}\right), \quad \forall c, d \in \mathbb{R}, \quad q>1
$$

and $w_{1}$ is a solution of Eq.(1), we obtain that

$$
\begin{aligned}
I\left(s w_{1}-t v_{\varepsilon}\right) & \leq I\left(s w_{1}\right)+I\left(t v_{\varepsilon}\right)-s t \int\left(\left|w_{1}\right|^{p-1} v_{\varepsilon}+f v_{\varepsilon}\right) \\
& +K_{4}\left(\int\left|s w_{1}\right|^{p-1}\left|t v_{\varepsilon}\right|+\int\left|s w_{1}\right|\left|t v_{\varepsilon}\right|^{p-1}\right) \\
& \leq I\left(s w_{1}\right)+I\left(t v_{\varepsilon}\right)+K_{5} \int\left(\left|w_{1}\right|^{p-1}\left|v_{\varepsilon}\right|+\left|w_{1}\right|\left|v_{\varepsilon}\right|^{p-1}+f v_{\varepsilon}\right) .
\end{aligned}
$$

Since $w_{1} \in \mathscr{M}$, we have that $I\left(s w_{1}\right) \leq I\left(w_{1}\right)$ for all $s \geq 0$. Note that

$$
\sup _{t \in \mathbb{R}} I\left(t v_{\varepsilon}\right) \leq \frac{1}{N} S_{\lambda}^{\frac{N}{2}}-O\left(\varepsilon^{\frac{\alpha \sqrt{\Lambda}}{2 \sqrt{\Lambda-\lambda}}}\right)+O\left(\varepsilon^{\frac{N-2}{4}}\right) .
$$

We obtain from Proposition 2.4 that

$$
\begin{aligned}
& \max _{s>0, t \in \mathbb{R}} I\left(s w_{1}-t v_{\varepsilon}\right) \\
& \leq \max _{s>0} I\left(s w_{1}\right)+\max _{t \in \mathbb{R}} I\left(t u_{\varepsilon}\right)+K_{7} \varepsilon^{(N-2) / 4}+K_{8} \varepsilon^{(N-2) / 4} \\
& \leq I\left(w_{1}\right)+\frac{1}{N} S_{\mu}^{\frac{N}{2}}-O\left(\varepsilon^{\frac{\alpha \sqrt{\Lambda}}{2 \sqrt{\Lambda-\lambda}}}\right)+K_{9} \varepsilon^{(N-2) / 4} \\
& <c_{1}+\frac{1}{N} S_{\mu}^{\frac{N}{2}},
\end{aligned}
$$

since $0<\alpha<\sqrt{\Lambda-\lambda}$. 
Remark 3.12 Similar to those pointed out in Remark 3.6, we emphasize that the exact local behavior of the solution of Eq.(1) (see Propositions 2.2 and 2.4) played an essential role in estimate of $c_{2}$.

Theorem 3.13 Let $0 \leq \lambda<\Lambda$ and $0<\alpha<\sqrt{\Lambda-\lambda}$ and (A) holds. Then there is a $w_{2} \in \mathscr{M}_{*}^{-}$such that $I\left(w_{2}\right)=c_{2}$ and $w_{2}$ is a sign changing solution of Eq.(1).

Proof. In the first step, we will prove that there is $w_{2} \in \mathscr{M}_{*}^{-}$such that $I\left(w_{2}\right)=c_{2}$. Let $\left\{u_{n}\right\} \subset \mathscr{M}_{*}^{-}$be such that $I\left(u_{n}\right) \rightarrow c_{2}$. Using the fact that $\left\{u_{n}^{+}\right\} \subset \mathscr{M}^{-}$and Sobolev inequality, one can easily show that

$$
0<\inf \left\|u_{n}^{+}\right\| \leq \sup \left\|u_{n}^{+}\right\|<+\infty
$$

Similarly we have that $\left\{u_{n}^{-}\right\}$is bounded with respect to $n$. Going if necessary to a subsequence, we may assume that $u_{n}^{+} \rightarrow u^{+}$and $u_{n}^{-} \rightarrow u^{-}$in $H_{0}^{1}(\Omega)$ and that $I\left(u_{n}^{+}\right) \rightarrow d_{1}, I\left(u_{n}^{-}\right) \rightarrow d_{2}$ and $c_{2}=d_{1}+d_{2}$. We claim that $u^{+} \neq 0$ and $u^{-} \neq 0$. If $u^{+}=0$ and $u^{-}=0$, then by Proposition 3.3, $d_{1} \geq \frac{1}{N} S_{\lambda}^{\frac{N}{2}}$, $d_{2} \geq \frac{1}{N} S_{\lambda}^{\frac{N}{2}}$ and hence $c_{2} \geq \frac{2}{N} S_{\lambda}^{\frac{N}{2}}$. If $u^{+}=0$ and $u^{-} \neq 0$, then by Proposition $3.3, d_{1} \geq \frac{1}{N} S_{\lambda}^{\frac{N}{2}}, d_{2} \geq c_{1}$ or $d_{2} \geq c_{0}+\frac{1}{N} S_{\lambda}^{\frac{N}{2}}$, which implies that $c_{2} \geq c_{1}+\frac{1}{N} S_{\lambda}^{\frac{N}{2}}$ or $c_{2} \geq c_{0}+\frac{2}{N} S_{\lambda}^{\frac{N}{2}}$. If $u^{+} \neq 0$ and $u^{-}=0$, then by Proposition 3.3, one gets $c_{2} \geq c_{1}+\frac{1}{N} S_{\lambda}^{\frac{N}{2}}$ or $c_{2} \geq c_{0}+\frac{2}{N} S_{\lambda}^{\frac{N}{2}}$. All the above three cases contradict Lemma 3.5 and Lemma 3.11. Therefore $u^{+} \neq 0$ and $u^{-} \neq 0$. According to (1) and (2) of Proposition 3.3, we have one of the following:

(i) $\left\{u_{n}^{+}\right\}$converges strongly to $u^{+}$;

(ii) $d_{1}>I\left(t^{+}\left(u^{+}\right) u^{+}\right)$;

(iii) $d_{1}>I\left(t^{-}\left(u^{+}\right) u^{+}\right)+\frac{1}{N} S_{\lambda}^{\frac{N}{2}}$;

and we also have one of the following:

(iv) $\left\{u_{n}^{-}\right\}$converges strongly to $u^{-}$;

(v) $d_{2}>I\left(-t^{+}\left(-u^{-}\right) u^{-}\right)$;

(vi) $d_{2}>I\left(-t^{-}\left(-u^{-}\right) u^{-}\right)+\frac{1}{N} S_{\lambda}^{\frac{N}{2}}$.

We will prove that only cases (i) and (iv) hold. For example, in the case (ii) and (v), we have that $t^{+}\left(u^{+}\right) u^{+}-t^{+}\left(-u^{-}\right) u^{-} \in \mathscr{M}_{*}^{-}$and hence

$c_{2} \leq I\left(t^{+}\left(u^{+}\right) u^{+}-t^{+}\left(-u^{-}\right) u^{-}\right)=I\left(t^{+}\left(u^{+}\right) u^{+}\right)+I\left(-t^{+}\left(-u^{-}\right) u^{-}\right)<d_{1}+d_{2}=c_{2}$, 
which is a contradiction. In the case (iii) and (vi), we have that $t^{-}\left(u^{+}\right) u^{+}-$ $t^{-}\left(-u^{-}\right) u^{-} \in \mathscr{M}^{+}$and hence

$$
\begin{aligned}
c_{1}+\frac{1}{N} S_{\lambda}^{\frac{N}{2}} & <c_{0}+\frac{2}{N} S_{\lambda}^{\frac{N}{2}} \leq I\left(t^{-}\left(u^{+}\right) u^{+}-t^{-}\left(-u^{-}\right) u^{-}\right)+\frac{2}{N} S_{\lambda}^{\frac{N}{2}} \\
& =I\left(t^{-}\left(u^{+}\right) u^{+}\right)+I\left(-t^{-}\left(u^{-}\right) u^{-}\right)+\frac{2}{N} S_{\lambda}^{\frac{N}{2}} \\
& \leq d_{1}+d_{2}=c_{2},
\end{aligned}
$$

which contradicts to Lemma 3.11. In the cases (ii) and (vi), we have that $t^{+}\left(u^{+}\right) u^{+}-t^{-}\left(-u^{-}\right) u^{-} \in \mathscr{M}^{-}$and hence

$$
c_{1}+\frac{1}{N} S_{\lambda}^{\frac{N}{2}} \leq I\left(t^{+}\left(u^{+}\right) u^{+}+t^{-}\left(u^{-}\right) u^{-}\right)+\frac{1}{N} S_{\lambda}^{\frac{N}{2}}<d_{1}+d_{2}=c_{2},
$$

which again contradicts to Lemma 3.11. In the case (i) and (v), we have $u^{+}-t^{+}\left(-u^{-}\right) u^{-} \in \mathscr{M}_{*}^{-}$and hence

$$
c_{2} \leq I\left(u^{+}-t^{+}\left(-u^{-}\right) u^{-}\right)<d_{1}+d_{2}=c_{2},
$$

which is a contradiction. Therefore we prove that only cases (i) and (iv) hold. Hence both $\left\{u_{n}^{+}\right\}$and $\left\{u_{n}^{-}\right\}$converge strongly to $u^{+}$and $u^{-}$, respectively and we get that $u^{+}, u^{-} \in \mathscr{M}^{-}$. Denote $w_{2}=u^{+}-u^{-}$. We get that $I\left(w_{2}\right)=c_{2}$.

Next we show that $w_{2}$ is a critical point of $I$. Suppose that $w_{2}$ is not a critical point of $I$, i.e. $\nabla I\left(w_{2}\right) \neq 0$. Note that for $u \in \mathscr{M}^{-}$, we have that

$$
\langle\nabla Q(u), u\rangle=J(u)<0 .
$$

Hence we can define

$$
V(u)=\nabla I(u)-\left\langle\nabla I(u), \frac{\nabla Q(u)}{\|\nabla Q(u)\|}\right\rangle \frac{\nabla Q(u)}{\|\nabla Q(u)\|}, \quad u \in \mathscr{M}^{-} .
$$

Choosing $\delta \in\left(0, \min \left\{\left\|u^{+}\right\|,\left\|u^{-}\right\|\right\} / 3\right)$ such that $\left\|V(v)-V\left(w_{2}\right)\right\| \leq \frac{1}{2}\left\|V\left(w_{2}\right)\right\|$ for each $v \in \mathscr{M}^{-}$with $\left\|v-w_{2}\right\| \leq 2 \delta$. Let $\psi: \mathscr{M}^{-} \rightarrow[0,1]$ be a Lipschitz mapping such that

$$
\psi(v)=\left\{\begin{array}{lll}
1 & \text { for } v \in \mathscr{M}^{-} \quad \text { with } & \left\|v-w_{2}\right\| \leq \delta, \\
0 & \text { for } v \in \mathscr{M}^{-} \quad \text { with } & \left\|v-w_{2}\right\| \geq 2 \delta .
\end{array}\right.
$$

Let $\eta:\left[0, s_{0}\right] \times \mathscr{M}^{-}$be the solution of the differential equation

$$
\eta(0, v)=v, \quad \frac{d}{d s} \eta(s, v)=-\psi(\eta(s, v)) V(\eta(s, v)) \quad \text { for } \quad(s, v) \in\left[0, s_{0}\right] \times \mathscr{M}^{-},
$$

where $s_{0}$ is some positive number. We set

$\chi(t)=t^{+}\left((1-t) u^{+}-t u^{-}\right) \cdot\left((1-t) u^{+}-t u^{-}\right) \quad$ and $\quad \xi(t)=\eta\left(s_{0}, \chi(t)\right)$ for $0 \leq t \leq 1$. 
Keep the definition of $u^{+}=\max \{u, 0\}$ and $u^{-}=\max \{-u, 0\}$ in mind. We have that if $t \in\left(0, \frac{1}{2}\right) \cup\left(\frac{1}{2}, 1\right)$, then

$$
I(\xi(t)) \leq I(\chi(t))=I\left(\chi(t)^{+}\right)+I\left(\chi(t)^{-}\right)<I\left(u^{+}\right)+I\left(u^{-}\right)=I\left(w_{2}\right)
$$

and $I\left(\xi\left(\frac{1}{2}\right)\right)<I\left(\chi\left(\frac{1}{2}\right)\right)=I\left(w_{2}\right)$. Therefore $I(\xi(t))<I\left(w_{2}\right)$ for $t \in(0,1)$. Since $t^{+}\left(\xi(t)^{+}\right)-t^{+}\left(-\xi(t)^{-}\right) \rightarrow-\infty$ as $t \rightarrow 0+$ and $t^{+}\left(\xi(t)^{+}\right)-t^{+}\left(-\xi(t)^{-}\right) \rightarrow+\infty$ as $t \rightarrow 1-0$, we get a $t_{1} \in(0,1)$ such that $t^{+}\left(\xi\left(t_{1}\right)^{+}\right)=t^{+}\left(-\xi\left(t_{1}\right)^{-}\right)$. So $\xi\left(t_{1}\right)=\xi\left(t_{1}\right)^{+}-\xi\left(t_{1}\right)^{-} \in \mathscr{M}_{*}^{-}$and $I\left(\xi\left(t_{1}\right)\right)<I\left(w_{2}\right)$, which is a contradiction. Hence we obtain $\nabla I\left(w_{2}\right)=0$.

\subsection{A fourth solution}

Up to now, we got three solutions $w_{0}, w_{1}$ and $w_{2}$. Next we will prove that there is another solution by a translated argument. We define a $C^{1}$ functional $\bar{I}: H_{0}^{1}(\Omega) \rightarrow \mathbb{R}$ by

$$
\bar{I}(v) \doteq \frac{1}{2} T(v)-\frac{1}{p} \int\left(\left|v^{+}+w_{0}\right|^{p}-\left|w_{0}\right|^{p}-p\left|w_{0}\right|^{p-2} w_{0} v^{+}\right)
$$

for $v \in H_{0}^{1}(\Omega)$. Consider the following minimax value

$$
\bar{c} \doteq \inf _{\gamma \in \Gamma} \sup _{0 \leq t \leq 1} \bar{I}(\gamma(t))
$$

where

$$
\Gamma \doteq\left\{\gamma \in C\left([0,1], H_{0}^{1}(\Omega)\right): \gamma(0)=0, \gamma(1)=k v_{\varepsilon}\right\}
$$

with suitable $\varepsilon$ and $k$.

Lemma 3.14 We have $\bar{c}<\frac{1}{N} S_{\lambda}^{\frac{N}{2}}$.

Proof. The proof is almost the same as Chen [5, Lemma 5.2].

Lemma $3.15 \bar{I}$ satisfies the $(P S)_{c}$ condition for $\bar{c}<\frac{1}{N} S_{\lambda}^{\frac{N}{2}}$.

Proposition 3.16 There exists a critical point $\bar{w}_{1} \in H_{0}^{1}(\Omega)$ of $I$ such that $\bar{w}_{1}>w_{0}$ in $\Omega$. Moreover, $w_{2} \neq \bar{w}_{1}$.

Proof. Similar to those in the proof of Chen [5, Lemma 5.1], we know that 0 is a local minimizer of $\bar{I}$. By Lemmas 3.14 and 3.15 and a standard mountain pass theorem (see Rabinowitz [12], Struwe [14], Willem [18]), we obtain that there is a critical point $v \neq 0$ of $\bar{I}$. By standard argument and maximum 
principle we have that $v>0$ in $\Omega$. Set $\bar{w}_{1}=v+w_{0}$. Then $\bar{w}_{1}$ is is a critical point of $I$ and $\bar{w}_{1}>w_{0}$ in $\Omega$.

Next we show that $w_{2} \neq \bar{w}_{1}$. Suppose that $w_{2}=\bar{w}_{1}$. Then we have that $0 \geq-w_{2}^{-} \geq-w_{0}^{-}$. Since $-w_{2}^{-} \in \mathscr{M}^{-}$and $w_{0} \in \mathscr{M}^{+}$, we get that

$T\left(-w_{2}^{-}\right)<(p-1) \int\left|-w_{2}^{-}\right|^{p} \leq(p-1) \int\left|-w_{0}^{-}\right|^{p} \leq(p-1) \int\left|w_{0}\right|^{p}<T\left(w_{0}\right)$.

On the other hand, using Lemma 3.1 and assumption (A),

$$
T\left(w_{0}\right)<\left(\frac{p-1}{p-2} M^{-1}|f|_{\sharp}\right)^{2}<\left(\frac{1}{2}\left(\frac{M^{p}}{p-1}\right)^{\frac{1}{p-2}}\right)^{2}<T\left(-w_{2}^{-}\right),
$$

which is a contradiction. Thus, we have proved $w_{2} \neq \bar{w}_{1}$.

Proposition 3.17 There exists a critical point $\widehat{w}_{1} \in H_{0}^{1}(\Omega)$ of I such that $\widehat{w}_{1}<w_{0}$ in $\Omega$. Moreover, $w_{2} \neq \widehat{w}_{1}$.

Proof. For $v \in H_{0}^{1}(\Omega)$, we define the following functional

$$
\widehat{I}(v) \doteq \frac{1}{2} T(v)-\frac{1}{p} \int\left(\left|w_{0}-v^{-}\right|^{p}-\left|w_{0}\right|^{p}+p\left|w_{0}\right|^{p-2} w_{0} v^{-}\right) .
$$

Now using the same procedure as in getting the solution $\bar{w}_{1}$, we can easily get the existence of a critical point $\widehat{w}_{1} \in H_{0}^{1}(\Omega)$ of $I$ and $\widehat{w}_{1}$ satisfies all the requirement of Proposition 3.17.

Proof of Theorem 1.2. From the previous three subsection, we have got five weak solutions of Eq.(1) $w_{0}, w_{1}, w_{2}, \bar{w}_{1}$ and $\widehat{w}_{1}$. Since $w_{1}$ may equal to $\bar{w}_{1}$ or $\widehat{w}_{1}$, we have obtained at least four solutions $w_{0}, w_{2}, \bar{w}_{1}$ and $\widehat{w}_{1}$ of Eq.(1), and we know that $w_{2}$ is sign changing.

Acknowledgments. The authors acknowledge the partial financial support from NSF of China (No.10501006), NSF of Fujian (2008J0189) and the Programme of NCETFJ; and from the Portuguese Foundation for Science and Technology (FCT), under the fellowship SFRH/BPD/38436/2007, and the research unit Mathematics and Applications.

\section{References}

[1] H. Brezis and L. Nirenberg, Positive solutions of nonlinear elliptic equations involving critical exponents, Comm. Pure Appl. Math. 34(1983) 437-477. 
[2] N. Chaudhuri and M. Ramaswamy, Existence of positive solutions of some semilinear elliptic equations with singular coefficients, Proc. Roy. Soc. Edinburgh 131A(2001) 1275-1295.

[3] F. Catrina and Z.Q. Wang, On the Caffarelli-Kohn-Nirenberg inequalities: sharp constants, existence (and nonexistence) and symmetry of extremal functions, Comm. Pure. Appl. Math. 56(2001) 229-258.

[4] J. Chen, S.J. Li and Y.Q. Li, Multiple solutions for a semilinear equation involving singular potential and critical exponent, Z. angew. Math. Phys. 56(2005) 453-474.

[5] J. Chen, Multiple positive solutions for a class of nonlinear elliptic equation, J. Math. Anal. Appl. 295(2004) 341-354.

[6] J. Chen, On a semilinear elliptic equation with singular term and HardySobolev critical growth, Math. Nachr. 280(2007) 838-850.

[7] K.S. Chou and C.W. Chu, On the best constant for a weighted SobolevHardy inequality, J. London Math. Soc. 48(1993) 137-151.

[8] A. Ferrero and F. Gazzola, Existence of solutions for singular critical growth semilinear elliptic equations, J. Differential Equations 177(2001) $494-522$.

[9] N. Hirano and N. Shioji, A multiplicty result including a sign changing solution for an inhomogeneous Neumann problem with critical exponent, Proc. Roy. Soc. Edinburgh 137A(2007) 333-347.

[10] E. Jannelli, The role played by space dimension in elliptic critical problems, J. Differential Equations 156(1999) 407-426.

[11] D. Kang and Y. Deng, Multiple solutions for inhomogeneous elliptic problems involving critical Sobolev-Hardy exponents, J. Math. Anal. Appl. 60(2005) 729-753.

[12] P. Rabinowitz, Minimax Methods in Critical Points Theory with Applications to Differential Equation, CBMS series 65, Providence R.I., 1986.

[13] D. Smets, Nonlinear Schrödinger equations with Hardy potential and critical nonlinearities, Trans. Amer. Math. Soc. 357(2005) 2909-2938.

[14] M. Struwe, Variational Methods, Applications to Nonlinear Partial Differential Equations and Hamiltonian Systems, Second Edition, SpringerVerlag, 1996.

[15] G. Tarantello, Multiplicity results for an inhomogeneous Neumann problem with critical exponent, Manuscripta Math. 81(1993) 51-78.

[16] G. Tarantello, On nonhomogeneous elliptic equations involving critical Sobolev exponent, Ann. Inst. H. Poincaré Anal. Non Linéaire 9(1992) 281-309.

[17] S. Terracini, On positive entire solutions to a class of equations with singular coefficient and critical exponent, Adv. Differential Equations 1(1996), 241-264.

[18] M. Willem, Minimax Theorems, Birkhäuser, Boston, 1996. 